\title{
AveBoost2: Boosting for Noisy Data
}

\author{
Nikunj C. Oza \\ Computational Sciences Division \\ NASA Ames Research Center \\ Mail Stop 269-2 \\ Moffett Field, CA 94035-1000, USA \\ oza@email.arc.nasa.gov
}

\begin{abstract}
AdaBoost [4] is a well-known ensemble learning algorithm that constructs its constituent or base models in sequence. A key step in AdaBoost is constructing a distribution over the training examples to create each base model. This distribution, represented as a vector, is constructed to be orthogonal to the vector of mistakes made by the previous base model in the sequence [6]. The idea is to make the next base model's errors uncorrelated with those of the previous model. In previous work [8], we developed an algorithm, AveBoost, that constructed distributions orthogonal to the mistake vectors of all the previous models, and then averaged them to create the next base model's distribution. Our experiments demonstrated the superior accuracy of our approach. In this paper, we slightly revise our algorithm to allow us to obtain non-trivial theoretical results: bounds on the training error and generalization error (difference between training and test error). Our averaging process has a regularizing effect which, as expected, leads us to a worse training error bound for our algorithm than for AdaBoost but a superior generalization error bound. For this paper, we experimented with the data that we used in [8] both as originally supplied and with added label noise - a small fraction of the data has its original label changed. Noisy data are notoriously difficult for AdaBoost to learn. Our algorithm's performance improvement over AdaBoost is even greater on the noisy data than the original data.
\end{abstract}

\section{Introduction}

AdaBoost [4] is one of the most well-known and highest-performing ensemble classifier learning algorithms [3]. It constructs a sequence of base models, where each model is constructed based on the performance of the previous model on the training set. In particular, AdaBoost calls the base model learning algorithm with a training set weighted by a distribution. ${ }^{1}$ After the base model is created, it is tested on the training set to see how well it learned. We assume that the base model learning algorithm is a weak learning algorithm [5]; that is, with

\footnotetext{
${ }^{1}$ If the base model learning algorithm cannot take a weighted training set as input, then one can create a sample with replacement from the original training set according to the distribution and call the algorithm with that sample.
} 
high probability, it produces a model whose probability of misclassifying an example is less than 0.5 when that example is drawn from the same distribution that generated the training set. The point is that such a model performs better than random guessing. ${ }^{2}$ The weights of the correctly classified examples and misclassified examples are scaled down and up, respectively, so that the two groups' total weights are 0.5 each. The next base model is generated by calling the learning algorithm with this new weight distribution and the training set. The idea is that, because of the weak learning assumption, at least some of the previously misclassified examples will be correctly classified by the new base model. Previously misclassified examples are more likely to be classified correctly because of their higher weights, which focus more attention on them. Kivinen and Warmuth [6] have shown that AdaBoost scales the distribution with the goal of making the next base model's mistakes uncorrelated with those of the previous base model.

AdaBoost is notorious for performing poorly on noisy datasets [3], such as those having some examples that were assigned the wrong class label. Because these examples are inconsistent with the majority of examples, they tend to be harder for the base model learning algorithm to learn. AdaBoost increases the weights of examples that the base model learning algorithm did not learn correctly. Noisy examples are likely to be incorrectly learned by many of the base models so that eventually these examples' weights will dominate those of the remaining examples. This causes AdaBoost to focus too much on the noisy examples at the expense of the majority of the training examples, leading to poor performance on new examples.

We previously [8] presented an algorithm, called AveBoost, which calculates the next base model's distribution by first calculating a distribution the same way as in AdaBoost, but then averaging it elementwise with those calculated for the previous base models. This averaging mitigates AdaBoost's tendency to increase the weights of noisy examples to excess. In our previous work we presented promising experimental results. However, we did not present theoretical results. We were unable to derive a non-trivial training error bound for the algorithm presented in [8]. In this paper, we present a slight modification to A veBoost which allows us to obtain both a non-trivial training error bound and a generalization error bound (difference between training error and test error). We call this algorithm AveBoost2. In Section 2, we review AdaBoost. In Section 3, we describe the AveBoost 2 algorithm and state our modification to what we presented previously. In Section 4, we present our training error bound and generalization error bound. The averaging in our algorithm has a regularizing effect; therefore, as expected, our training error bound is worse than that of AdaBoost but our generalization error bound is better than AdaBoost's. In Section 5, we present an experimental comparison of our new AveBoost2 with AdaBoost on

${ }^{2}$ The version of AdaBoost that we use was designed for two-class classification problems. However, it is often used for a larger number of classes when the base model learning algorithm is strong enough to have an error less than 0.5 in spite of the larger number of classes. 
$\operatorname{AdaBoost}\left(\left\{\left(x_{1}, y_{1}\right), \ldots,\left(x_{m}, y_{m}\right)\right\}, L_{b}, T\right)$

Initialize $d_{1, i}=1 / m$ for all $i \in\{1,2, \ldots, m\}$.

For $t=1,2, \ldots, T$ :

$h_{t}=L_{b}\left(\left\{\left(x_{1}, y_{1}\right), \ldots,\left(x_{m}, y_{m}\right)\right\}, \mathbf{d}_{t}\right)$.

Calculate the error of $h_{t}: \epsilon_{t}=\sum_{i: h_{t}\left(x_{i}\right) \neq y_{i}} d_{t, i}$.

If $\epsilon_{t} \geq 1 / 2$ then, set $T=t-1$ and abort this loop.

$\beta_{t}=\frac{\epsilon_{t}}{1-\epsilon_{t}}$.

Calculate distribution $\mathrm{d}_{t+1}$ :

$$
\begin{aligned}
w_{i} & =d_{t, i} \times \begin{cases}\beta_{t} \text { if } h_{t}\left(x_{i}\right)=y_{i} \\
1 & \text { otherwise. }\end{cases} \\
d_{t+1, i} & =\frac{w_{i}}{\sum_{i=1}^{m} w_{i}}
\end{aligned}
$$

Output the final hypothesis:

$$
h_{f i n}(x)=\operatorname{argmax}_{y \in Y} \sum_{t: h_{t}(x)=y} \log \frac{1}{\beta_{t}} .
$$

Fig. 1. AdaBoost algorithm: $\left\{\left(x_{1}, y_{1}\right), \ldots,\left(x_{m}, y_{m}\right)\right\}$ is the training set, $L_{b}$ is the base model learning algorithm, and $T$ is the maximum allowed number of base models.

some UCI datasets [1] both in original form and with $10 \%$ label noise added. Section 6 summarizes this paper and describes ongoing and future work.

\section{AdaBoost}

Figure 1 shows AdaBoost's pseudocode. AdaBoost constructs a sequence of base models $h_{t}$ for $t \in\{1,2, \ldots, T\}$, where each model is constructed based on the performance of the previous base model on the training set. In particular, AdaBoost maintains a distribution over the $m$ training examples. The distribution $\mathbf{d}_{1}$ used in creating the first base model gives equal weight to each example $\left(d_{1, i}=1 / m\right.$ for all $\left.i \in\{1,2, \ldots, m\}\right)$. AdaBoost now enters the loop, where the base model learning algorithm $L_{b}$ is called with the training set and $d_{1} \cdot{ }^{3}$ The returned model $h_{1}$ is then tested on the training set to see how well it learned. The total weight of the misclassified examples $\left(\epsilon_{1}\right)$ is calculated. The weights of the correctly-classified examples are multiplied by $\epsilon_{1} /\left(1-\epsilon_{1}\right)$ so that they have the same total weight as the misclassified examples. The weights are then normalized so that they sum to 1 instead of $2 \epsilon_{1}$. AdaBoost assumes that $L_{b}$ is a weak learner, i.e., $\epsilon_{t}<\frac{1}{2}$ with high probability. Under this assumption, the total weight of the misclassified examples $\epsilon_{t}<1 / 2$ is increased to $1 / 2$ and the

${ }^{3}$ As mentioned earlier, if $L_{b}$ cannot take a weighted training set as input, then we can give it a sample drawn with replacement from the original training set according to the distribution $\mathbf{d}$ induced by the weights. 
$\operatorname{AveBoost2}\left(\left\{\left(x_{1}, y_{1}\right), \ldots,\left(x_{m}, y_{m}\right)\right\}, L_{b}, T\right)$

Initialize $d_{1, i}=1 / m$ for all $i \in\{1,2, \ldots, m\}$.

For $t=1,2, \ldots, T$ :

$h_{t}=L_{b}\left(\left\{\left(x_{1}, y_{1}\right), \ldots,\left(x_{m}, y_{m}\right)\right\}, \mathrm{d}_{t}\right)$.

Calculate the error of $h_{t}: \epsilon_{t}=\sum_{i: h_{t}\left(x_{i}\right) \neq y_{i}} d_{t, i}$.

If $\epsilon_{t} \geq 1 / 2$ then,

set $T=t-1$ and abort this loop.

$\beta_{t}=\frac{\epsilon_{t}}{1-\epsilon_{t}}$.

$\gamma_{t}=\frac{2\left(1-\epsilon_{t}\right) t+1}{2 \epsilon_{t} t+1}$.

Calculate orthogonal distribution:

For $i=1,2, \ldots, m$ :

$$
\begin{aligned}
w_{i} & =d_{t, i} \times \begin{cases}\beta_{t} & \text { if } h_{t}\left(x_{i}\right)=y_{i} \\
1 & \text { otherwise }\end{cases} \\
c_{t, i} & =\frac{w_{i}}{\sum_{i=1}^{m} w_{i}} . \\
d_{t+1, i} & =\frac{t d_{t, i}+c_{t, i}}{t+1}
\end{aligned}
$$

Output the final hypothesis:

$$
h_{f i n}(x)=\operatorname{argmax}_{y \in Y} \sum_{t: h_{t}(x)=y} \log \frac{1}{\beta_{t} \gamma_{t}} .
$$

Fig. 2. AveBoost2 algorithm: $\left\{\left(x_{1}, y_{1}\right), \ldots,\left(x_{m}, y_{m}\right)\right\}$ is the training set, $L_{b}$ is the base model learning algorithm, and $T$ is the maximum allowed number of base models.

total weight of the correctly classified examples $1-\epsilon_{t}>1 / 2$ is decreased to $1 / 2$. This is done so that, by the weak learning assumption, $h_{t+1}$ will classify at least some of the previously misclassified examples correctly. Returning to the algorithm, the loop continues, creating the $T$ base models in the ensemble. The final ensemble returns, for a new example, the one class in the set of classes $Y$ that gets the highest weighted vote from the base models.

\section{AveBoost2 algorithm}

Figure 2 shows our new algorithm, AveBoost2. Just as in AdaBoost, AveBoost2 initializes $d_{1, i}=1 / m$ for all $i \in\{1,2, \ldots, m\}$. Then it goes inside the loop, where it calls the base model learning algorithm $L_{b}$ with the training set and distribution $\mathbf{d}_{1}$ and calculates the error of the resulting base model $h_{1}$. It then calculates $\mathbf{c}_{1}$, which is the distribution that AdaBoost would use to construct the next base model. However, AveBoost 2 averages this with $\mathbf{d}_{1}$ to get $\mathbf{d}_{2}$, and uses this $\mathbf{d}_{2}$ instead. Showing that the $\mathbf{d}_{t}$ 's in AveBoost2 are distributions is a trivial proof by induction. For the base case, $\mathbf{d}_{1}$ is constructed to be a distribution. For the inductive part, if $\mathbf{d}_{t}$ is a distribution, then $\mathbf{d}_{t+1}$ is a distribution because it is 
a convex combination of $\mathbf{d}_{t}$ and $\mathbf{c}_{t}$, both of which are distributions. The vector $\mathbf{d}_{t+1}$ is a running average of $\mathbf{d}_{1}$ and the vectors $\mathbf{c}_{\mathbf{q}}$ for $q \in\{1,2, \ldots, t\}$.

Returning to the algorithm, the loop continues for a total of $T$ iterations. Then the base models are combined using a weighted voting scheme slightly different from that of AdaBoost and the original AveBoost from [8]: each model's weight is $\log \left(1 /\left(\beta_{t} \gamma_{t}\right)\right)$ instead of $\log \left(1 / \beta_{t}\right)$. AveBoost2 is actually AdaBoost with $\beta_{t}$ replaced by $\beta_{t} \gamma_{t}$. However, we wrote the AveBoost2 pseudocode as we did to make the running average calculation of the distribution explicit.

AveBoost2 can be seen as a relaxed version of AdaBoost. When training examples are noisy and therefore difficult to fit, AdaBoost is known to increase the weights on those examples to excess and overfit them [3] because many consecutive base models may not learn them properly. AveBoost2's averaging does not allow the weights of noisy examples to increase rapidly, thereby mitigating the overfitting problem. We therefore expect AveBoost2 to outperform AdaBoost on the noisy datasets to a greater extent than on the original datasets.

\section{Theory}

In this section, we give bounds on the training error and generalization error (difference between training and test error). Not surprisingly, the relaxed nature of AveBoost2 relative to AdaBoost caused us to obtain a worse training error bound but superior generalization error bound for AveBoost2 relative to AdaBoost. Due to space limitations, we defer the proofs and more intuition on the theoretical frameworks that we use to a longer version of this paper.

Theorem 1. In AveBoost2, suppose the weak learning algorithm $L_{b}$ generates hypotheses with errors $\epsilon_{1}, \epsilon_{2}, \ldots, \epsilon_{T}$ where each $\epsilon_{t}<1 / 2$. Then the ensemble's error $\epsilon=\sum_{i: h_{f_{i n}}\left(x_{i}\right) \neq y_{i}} d_{1, i}$ is bounded as follows:

$$
\epsilon \leq \prod_{t=1}^{T} \frac{t+1}{\sqrt{t^{2}+\frac{t}{2 \epsilon_{t}\left(1-\epsilon_{t}\right)}+\frac{1}{4 \epsilon_{t}\left(1-\epsilon_{t}\right)}}}
$$

This bound is clearly non-trivial $(\epsilon \leq 1)$; but it is greater than that of AdaBoost [4]:

$$
\epsilon \leq 2^{T} \prod_{t=1}^{T} \sqrt{\epsilon_{t}\left(1-\epsilon_{t}\right)} .
$$

To derive our generalization error bound, we use the algorithmic stability framework of [7]. Intuitively, algorithmic stability is very similar to Breiman's notion of stability [2] - the more stable a learning algorithm is, the less of an effect changes to the training set have on the model returned. Clearly, the more stable the learning algorithm is, the smaller the difference between the training and test errors tends to be. We show that AveBoost2 is more stable than AdaBoost; 
therefore, the difference between the training and test errors is lower. We first give some preliminaries from [7] and then state our new result.

For the following, $\mathcal{X}$ is the space of possible inputs, $\mathcal{Y}=\{0,1\}$ is the set of possible labels, and $\mathcal{Z}=\mathcal{X} \times \mathcal{Y}$.

Definition 1 (Definition 2.5 from [7]). A learning algorithm is a process which takes as input a distribution $p$ on $\mathcal{Z}$ with finite support and outputs a function $f_{p}: \mathcal{X} \rightarrow[0,1]$. For $S \in \mathcal{Z}^{m}$ for some positive integer $m, f_{S}$ means $f_{p}$ where $p$ is the uniform distribution on $S$.

In the following, the error of $f$ on an example $(x, y)$ is $c(f,(x, y))=|f(x)-y|$.

Definition 2 (Definition 2.11 from [7]). A learning algorithm has $L_{1}$-stability $\lambda$ if, for any two distributions $p$ and $q$ on $\mathcal{X}$ with finite support,

$$
\forall z \in \mathcal{Z},\left|c\left(f_{p}, z\right)-c\left(f_{q}, z\right)\right| \leq \lambda\|p-q\|_{1} .
$$

In the following, $D$ is a distribution on $\mathcal{Z}, S \sim D^{m}$ is a set of $m$ examples drawn from $\mathcal{Z}$ according to $D$, and $S^{i, u}$ is $S$ with example $i \in\{1,2, \ldots, m\}$ removed (each $i$ is chosen with probability $1 / m$ ) and example $u \sim D$ added.

Definition 3 (Definition 2.14 from [7]). A learning algorithm is $(\beta, \delta)$-stable if

$$
P_{S \sim D^{m}}\left(\left|c\left(f_{S}, z\right)-c\left(f_{S^{i, u}}, z\right)\right| \leq \beta\right) \geq 1-\delta .
$$

Intuitively, $f_{S}$ and $f_{S^{i, u}}$ are models that result from running the learning algorithm on two slightly different training sets. As $\beta$ and $\delta$ decrease, the probability of having smaller differences in errors between these two models increases, which means that the learning algorithm is more stable. Greater stability implies lower generalization error according to the following theorem. In the following, $\operatorname{Err}_{S}\left(f_{S}\right)$ is the training error (error on the training set $S$ ) and $\operatorname{Err}_{D}\left(f_{S}\right)$ is the test error, i.e., the error on an example $(x, y)$ chosen at random according to distribution $D$.

Theorem 2 (Theorem 3.4 from [7]). Suppose a $(\beta, \delta)$-stable learning algorithm returns a hypothesis $f_{S}$ for any training set $S \sim D^{m}$. Then for all $\tau>0$ and $m \geq 1$,

$P_{S \sim D^{m}}\left(\left|\operatorname{Err}_{S}\left(f_{S}\right)-\operatorname{Err}_{D}\left(f_{S}\right)\right|>\tau+\beta+\delta\right) \leq 2 \exp \left(\frac{-\tau^{2} m}{2(2 m \beta+1)^{2}}\right)+\frac{4 m^{2} \delta}{2 m \beta+1}$.

Intuitively, this theorem shows that lower values of $\beta$ and $\delta$ lead to lower probabilities of large differences between the training and test errors. We can finally state our theorem on AveBoost2's stability.

Theorem 3. Suppose the base model learning algorithm has $L_{1}$-stability $\lambda$ and $\min _{t \in\{1,2, \ldots, T\}} \epsilon_{t}>\epsilon_{*}>0$. Then, for sufficiently large $m$ and for all $T$, AveBoost2 is $(\beta, \delta)$-stable, where 
Table 1. The datasets used in the experiments

\begin{tabular}{|c|c|c|c|c|}
\hline Data Set & $\begin{array}{c}\text { Training } \\
\text { Set }\end{array}$ & $\begin{array}{c}\text { Test } \\
\text { Set }\end{array}$ & Inputs & Classes \\
\hline \hline Promoters & 84 & 22 & 57 & 2 \\
\hline Balance & 500 & 125 & 4 & 3 \\
\hline Breast Cancer & 559 & 140 & 9 & 2 \\
\hline German Credit & 800 & 200 & 20 & 2 \\
\hline Car Evaluation & 1382 & 346 & 6 & 4 \\
\hline Chess & 2556 & 640 & 36 & 2 \\
\hline Mushroom & 6499 & 1625 & 22 & 2 \\
\hline Nursery & 10368 & 2592 & 8 & 5 \\
\hline Connect4 & 54045 & $\mathbf{1 3 5 1 2}$ & 42 & 3 \\
\hline
\end{tabular}

Table 2. Performance of AveBoost2 compared to AdaBoost

\begin{tabular}{|c|c|c|c||c|c|c|c|}
\hline ORIGINAL & \multicolumn{2}{|c|}{ Num. Base Models } & $10 \%$ NOISE & \multicolumn{2}{|c|}{ Num. Base Models } \\
\hline Base Model & 10 & 50 & 100 & Base Model & 10 & 50 & 100 \\
\hline \hline Naive Bayes & $+4=4-1$ & $+4=4-1$ & $+4=4-1$ & Naive Bayes & $+8=1-0$ & $+8=1-0$ & $+7=2-0$ \\
Decision Trees & $+2=6-1$ & $+1=6-2$ & $+2=6-1$ & Decision Trees & $+6=2-1$ & $+5=3-1$ & $+6=2-1$ \\
Decision Stumps & $+1=6-2$ & $+1=6-2$ & $+1=6-2$ & Decision Stumps & $+0=7-2$ & $+1=7-1$ & $+1=7-1$ \\
\hline
\end{tabular}

$$
\begin{aligned}
& \beta=\frac{2}{m} \sum_{t=1}^{T} 2^{3 t-2}\left(\frac{(\lambda+1) t}{\epsilon_{*}}\right)^{t} \\
& \delta=\exp \left(\frac{-m \epsilon_{*}^{2}}{2}\right)
\end{aligned}
$$

For AdaBoost, the theorem is the same except that [7]

$$
\beta=\frac{2}{m} \sum_{t=1}^{T} \frac{2^{t^{2}+1}(\lambda+1)^{t}}{\epsilon_{*}^{2 t-1}}
$$

which is clearly larger than the corresponding $\beta$ for AveBoost2. This means that AveBoost2's generalization error is less than that of AdaBoost by theorem 2.

\section{Experimental Results}

In this section, we compare AdaBoost and AveBoost2 on the nine UCI datasets [1] described in Table 1. We ran both algorithms with three different values of $T$, 


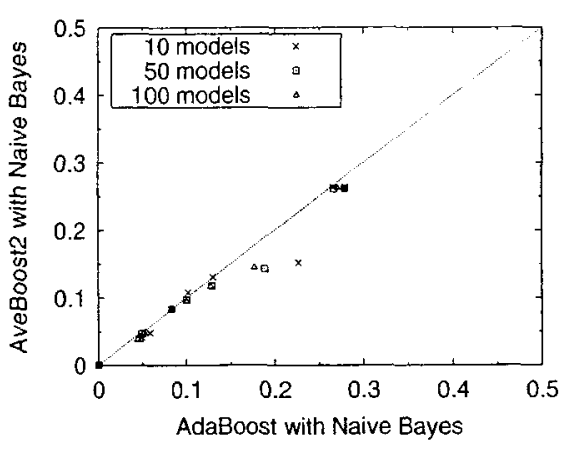

Fig. 3. Test set error rates of AdaBoost vs. AveBoost2 (Naive Bayes, original datasets)

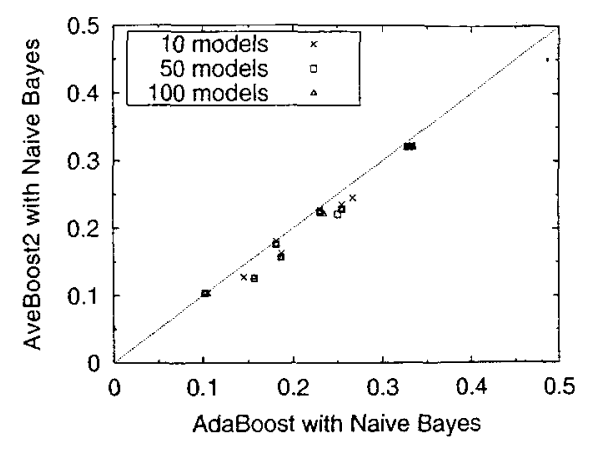

Fig. 4. Test set error rates of AdaBoost vs. AveBoost2 (Naive Bayes, noisy datasets)

which is the maximum number of base models that the algorithm is allowed to construct: 10,50, and 100. Each result reported is the average over 50 results obtained by performing 10 runs of 5 -fold cross-validation. Table 1 shows the sizes of the training and test sets for the cross-validation runs. We also repeated these runs after adding $10 \%$ label noise. That is, we randomly chose $10 \%$ of the examples in each dataset and changed their labels to one of the remaining labels with equal probability.

Table 2 shows how often AveBoost2 significantly outperformed, performed comparably with, and significantly underperformed AdaBoost. For example, on the original datasets and with 10 Naive Bayes base models, AveBoost2 significantly outperformed ${ }^{4}$ AdaBoost on four datasets, performed comparably on four datasets, and performed significantly worse on one, which is written as " $+4=4$ 1." Figures 3 and 4 compare the error rates of AdaBoost and AveBoost2 with Naive Bayes base models on the original and noisy datasets, respectively. In all the plots presented in this paper, each point marks the error rates of two algorithms when run with the number of base models indicated in the legend and a particular dataset. The diagonal lines in the plots contain points at which the two algorithms have equal error. Therefore, points below/above the line correspond to the error of the algorithm indicated on the $y$-axis being less than/greater than the error of the algorithm indicated on the $\mathrm{x}$-axis, respectively. We can see that, for Naive Bayes base models, AveBoost2 performs much better than AdaBoost overall, especially on the noisy datasets.

We compare AdaBoost and AveBoost2 using decision tree base models in figure 5 (original datasets) and figure 6 (noisy datasets). On the original datasets, the performances of the two algorithms are comparable. However, on the noisy datasets, AveBoost2 is superior for all except the Balance dataset. On the Balance dataset, AdaBoost actually performed as much as $10 \%$ better on the noisy data than the original data, which is strange, and needs to be investigated fur-

\footnotetext{
${ }^{4}$ We use a t-test with $\alpha=0.05$ to compare all the classifiers in this paper.
} 


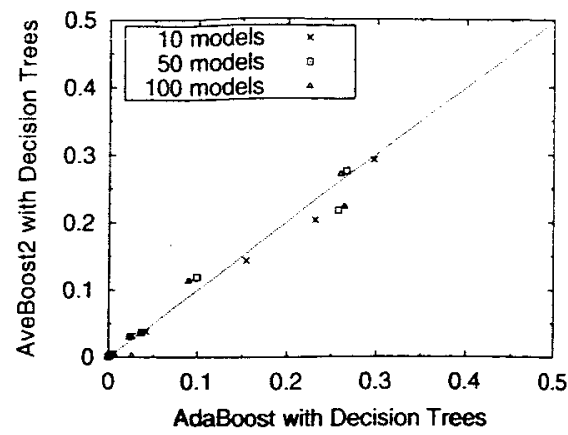

Fig. 5. Test set error rates of AdaBoost vs. AveBoost2 (Decision Trees, original datasets)

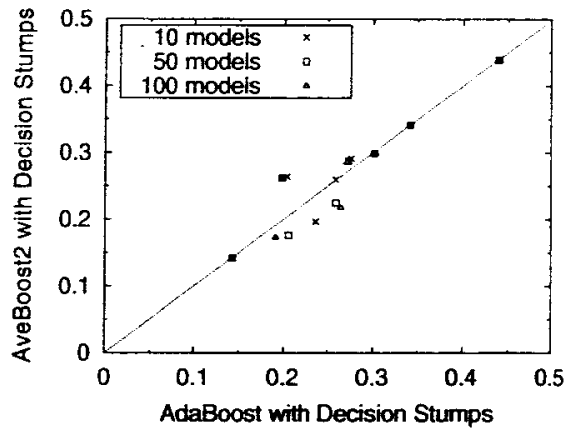

Fig. 7. Test set error rates of AdaBoost Fig. 8. Test set error rates of AdaBoost vs. AveBoost2 (Decision Stumps, original datasets)

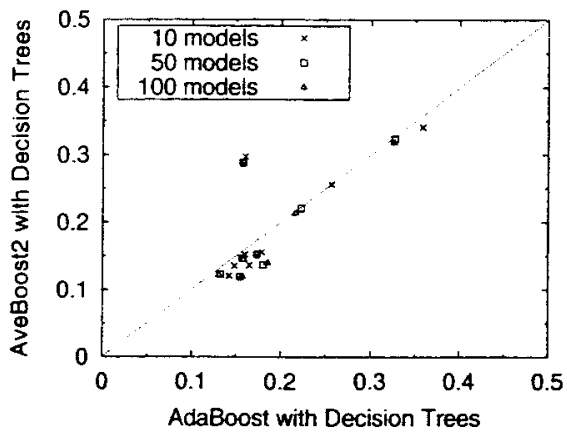

Fig. 6. Test set error rates of AdaBoost vs. AveBoost2 (Decision Trees, noisy datasets)

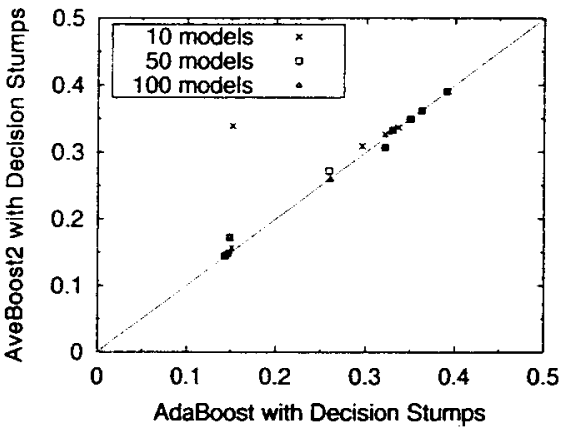

vs. AveBoost2 (Decision Stumps, noisy datasets)

ther. AveBoost2 performed worse on the noisy Balance data than on the original Balance data. Figure 7 gives the error rate comparison between AdaBoost and AveBoost2 with decision stump base models on the original datasets. Figure 8 gives the same comparison on the noisy datasets. With decision stumps, the two algorithms always seem to perform comparably. We suspect that decision stumps are too stable to allow the different distribution calculation methods of AdaBoost and AveBoost2 to yield significant differences.

\section{Conclusions}

We presented AveBoost2, a boosting algorithm that trains each base model using a training example weight vector that is based on the performances of all the previous base models rather than just the previous one. We discussed our 
theoretical results and demonstrated empirical results that are superior overall to AdaBoost; especially on datasets with label noise.

Our theoretical and empirical results do not account for what happens as the amount of noise changes. We plan to derive such results. In a longer version of this paper, we plan to perform a more detailed empirical analysis including the performances of the base models and ensembles on the training and test sets, correlations among the base models, ranges of the weights of regular and noisy examples, etc. In [8], we performed such an analysis to a limited extent for the original AveBoost and were able to confirm some of what we hypothesized there and in this paper: the base model accuracies tend to be higher than for AdaBoost, the correlations among the base models also tend to be higher, and the ranges of the weights of the training examples tends to be lower. We were unable to repeat this analysis here due to a lack of space. Such an analysis not only will enable us to understand empirically what is occurring but should guide our theoretical analysis of the performances of AdaBoost and AveBoost2 on noisy data.

\section{References}

1. C. Blake, E. Keogh, and C.J. Merz. UCI repository of machine learning databases, 1999. (URL: http://www.ics.uci.edu/ mlearn/MLRepository.html).

2. L. Breiman. Bagging predictors. Machine Learning, 24(2):123-140, 1996.

3. Thomas G. Dietterich. An experimental comparison of three methods for constructing ensembles of decision trees: Bagging, boosting, and randomization. Machine Learning, 40:139-158, Aug. 2000.

4. Y. Freund and R. Schapire. Experiments with a new boosting algorithm. In Proceedings of the Thirteenth International Conference on Machine Learning, pages 148-156, Bari, Italy, 1996. Morgan Kaufmann.

5. Michael J. Kearns and Umesh V. Vazirani. Introduction to Computational Learning Theory. MIT Press, Cambridge, MA, 1994.

6. Jyrki Kivinen and Manfred K. Warmuth. Boosting as entropy projection. In Proceedings of the Twelfth Annual Conference on Computational Learning Theory, pages 134-144, 1999.

7. Samuel Kutin and Partha Niyogi. The interaction of stability and weakness in adaboost. Technical Report TR-2001-30, University of Chicago, October 2001.

8. Nikunj C. Oza. Boosting with averaged weight vectors. In T. Windeatt and F. Roli, editors, Proceedings of the Fourth International Workshop on Multiple Classifier Systems, pages 15-24. Springer, Berlin, 2003. 